\title{
An experimental study on particle effects in liquid sheets
}

\author{
Alban Sauret ${ }^{1, \star}$, Anthony Troger $^{1}$, and Pierre Jop ${ }^{1}$ \\ ${ }^{1}$ Surface du Verre et Interfaces, UMR 125 CNRS/Saint-Gobain, 93303 Aubervilliers, France
}

\begin{abstract}
Many industrial processes, such as surface coating or liquid transport in tubes, involve liquid sheets or thin films of suspensions. In these situations, the thickness of the liquid film becomes comparable to the particle size, which leads to unexpected dynamics. In addition, the classical constitutive rheological law for suspensions cannot be applied as the continuum approximation is no longer valid. Here, we consider experimentally a transient particle-laden liquid sheet that expands radially. We characterize the influence of the particles on the shape of the liquid film and the atomization process. We highlight that the presence of particles modifies the thickness and stability of the liquid sheet. Our study suggests that the influence of particles through capillary effects can modify significantly the dynamics of processes that involve suspensions and particles confined in liquid films.
\end{abstract}

\section{Introduction}

Suspensions of solid non-brownian particles in a liquid are encountered in various geophysical, industrial or biological systems. Whereas most of the past studies have characterized the transport of particles in bulk flow or the rheology of suspensions [1,2], situations in which the thickness of the liquid layer becomes comparable to the particle size are less understood. This is particularly relevant in industrial processes, such as coating by liquid curtains. When the coating is performed with a suspension, the thickness and stability of the liquid sheet can be largely modified by the presence of particles [3]. Indeed, the particles are able to deform the liquid interface, which leads to complex interactions with the interfaces and between the particles.

The presence of particles in liquid film of comparable size can be especially important for its stability as it has been shown to modify the pinch-off of viscous fluid, when extruded from a nozzle to eventually generate a droplet. In a pure liquid, i.e., without particles, the liquid filament becomes thinner and thinner until the pinch off occurs owing to interfacial tension effects. Various studies have then considered the detachment of a droplet of granular suspension and have shown that the initial pinch-off can be described by its effective viscosity $[4,5]$. However, at later stages, the dynamics of the particles become important for the detachment of the drop when the liquid filament becomes of the same size as a particle. Typically, the detachment becomes accelerated and depends on the particle volume fraction and size. This uncommon situation cannot be explained with a classical Newtonian fluid approach or classical rheology of diluted suspensions. Indeed, the presence of particles perturbs locally the thinning of the filament. It then leads to the acceleration of the droplet

\footnotetext{
^e-mail: alban.sauret@gmail.com
}

formation compared to the detachment observed for a pure fluid.

However, the pinch-off is due to a $1 \mathrm{D}$ flow and more complex situations remain poorly understood. In particular, the influence of particles in self-suspended liquid films, which constitutes a 2D situation, could lead to a modification of its stability [3]. In this study, we consider experimentally a classical model system in which a drop impacts at the center of a flat target of size comparable to the drop diameter [6]. Upon impact the liquid flattens into a self-suspended liquid sheet that expands radially. Such experiments have been performed with Newtonian and non-Newtonian fluids to characterize the radial expansion of the liquid sheet and its fragmentation into droplets $[7,8]$ and with emulsions to investigate the influence of the liquid dispersed phase on the dynamics of the liquid sheet [9].

\section{Experimental methods and phenomenology}

The generation of the transient liquid sheet is based on a method adapted from the original experiments of Rozhkov et al. [6]. In our experimental set-up, shown in Fig. 1, a drop impacts the flat cross-section of a solid cylindrical target of diameter $d_{t}=6 \mathrm{~mm}$. The drop is formed by pushing the suspension through a needle positioned vertically above the target. The impact velocity $u_{0}$ on the target is directly set by the height of fall of the drop. The suspension is manually extruded from a syringe using a microcontroller linear stage to obtain monodisperse drops of diameter $d_{0}=3.9 \mathrm{~mm}$. The drop then falls on the target and spreads into a liquid sheet. A thin aluminium coaxial cylinder around the target keeps the angle of ejection of 

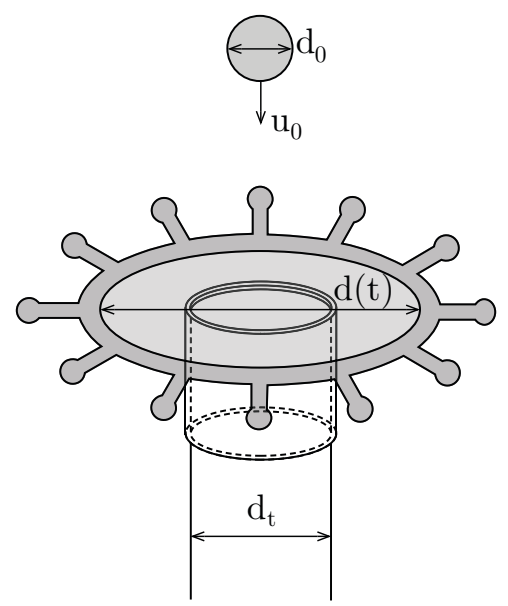

Figure 1. Schematic of the experimental set-up: a drop of diameter $d_{0}$ impacts the cylindrical target (diameter $d_{t}$ ) at a velocity $u_{0}$, leading to the formation of a transient liquid sheet of diameter $d(t)=2 R(t)$.

the liquid at $90^{\circ}$ and the liquid sheet horizontal. The target is mounted on a hollow plexiglas plate and illuminated from below using a LED Panel. We record the spreading of the sheet from the top using a high speed camera (Phantom v611) with a macro lens (Nikon $105 \mathrm{~mm}$ ) typically at $9000 \mathrm{fps}$.

The suspensions are prepared with spherical polystyrene PS particles (Dynoseeds) of density $\rho \simeq 1058 \mathrm{~kg} \mathrm{~m}^{3}$ dispersed in a mixture of $25 \% \mathrm{wt}$ glycerol/water solution such that the particles are neutrally buoyant. We use particles of diameter $d=140 \mu \mathrm{m}$ that are larger than the mean thickness of the liquid sheet. The viscosity of the glycerol/water mixture at $T=24^{\circ} \mathrm{C}$ is $\eta_{0}=1.85 \mathrm{mPa} . \mathrm{s}$ and its surface tension is $\gamma=70 \mathrm{mN} \cdot \mathrm{m}^{-1}$. The liquid viscosity is chosen small enough for the liquid sheet to reach a diameter of few centimeters. It requires that both viscous and capillary effects remain small compared to the inertial effects. In term of dimensionless number, this condition is described trough the Weber number

$$
W e=\frac{\rho u_{0}^{2} d_{0}}{\gamma},
$$

which compares the magnitude of inertial and capillary effects and the Capillary number

$$
C a=\frac{\eta u_{0}}{\gamma}
$$

which describes the ratio of viscous and capillary effects. Here, we have used a liquid phase with a low viscosity and work with diluted suspensions $(\phi=[0 \% ; 30 \%])$. Therefore, all of our experiments are performed in the regime where $W e \gg 1$ and $C a \ll 1$. The experiments presented in this paper are performed at a Weber number $W e \simeq 490$.

Two typical experiments, without and with particles, are shown in Fig. 2 and illustrate the dynamics of the liquid sheet from the impact of the drop on the target at $t=0 \mathrm{~ms}$ to the retraction and the atomization into droplets after about $10 \mathrm{~ms}$. We observe that the geometry of the liquid sheet during the spreading satisfies to the cylindrical symmetry of the geometry: a thin liquid sheet surrounded by a rim extends horizontally, suspended in air until it reaches the maximum diameter that results from a balance between the inertial and capillary effects. As shown on this figure, the value of the maximum diameter depends on the presence or absence of particles but also on the size of the drop and the impactor as well as the impact speed. Once the liquid sheet reaches its maximum diameter, the film retracts under the effect of capillary forces and destabilizes, losing its cylindrical symmetry. We also note that during most of the process, especially during the recessing phase, droplets are generated.

\section{Spreading of particle-laden liquid sheets}

We first characterized the evolution of the thickness of the liquid sheet in the absence of particles. We have implemented an optical method based on the absorption of light to measure the thickness of the sheet. This method relies on grayscale images of the sheet recorded with a highspeed camera. Using the Beer-Lambert law and a preliminary calibration, we can obtain the time and spatial evolution of the liquid thickness [10]. The results obtained via this method are shown in Fig. 3 and indicate that the thickness of the sheet varies between $20 \mu \mathrm{m}$ and $160 \mu \mathrm{m}$. The particles used in this study, of diameter $140 \mu \mathrm{m}$, are larger than the liquid thickness and will therefore be subject to capillary forces. We also observe that the thickness of the liquid sheet decreases with the distance $r$ from the center of the impactor at both short time and long time.

We consider the expansion dynamics of liquid sheet in the absence of particles to compare our results with previous predictions who considered the limit of long times and were able to show that the time evolution of the diameter of the liquid sheet is described by the expression [7]:

$$
\frac{R(t)-r_{0}}{r_{0}}=\sqrt{\frac{2}{3} W e} \frac{t}{\tau}\left(1-\frac{t}{\tau}\right)^{2}
$$

where $\tau=\sqrt{\rho d_{0}^{3} /(6 \gamma)}$. The spreading dynamics is partially in agreement with the theoretical modeling as shown in Fig. 4. In this model, we expect a prefactor $a=\sqrt{2 W e / 3} \simeq 18$, whereas our experiments suggest that $a=15$. Note that this discrepancy can be explained by the characteristics of the impactor, and in particular its wettability and the presence of the outer cylinder [10].

We have systematically studied the effect of the $140 \mu \mathrm{m}$ diameter particles on the spreading dynamic of the liquid sheet as shown in Fig. 4. The particles are larger than the liquid sheet thickness and are therefore subject to capillary force. We observe that, although the evolution are similar in all cases, there is a strong decrease of the maximum spreading diameter, even at low concentrations. We can also notice that the lifetime of the liquid sheet is greatly reduced by the presence of particles. More specifically, the expansion phase is slightly modified, but the total life is greatly reduced. A first assumption can be made to 


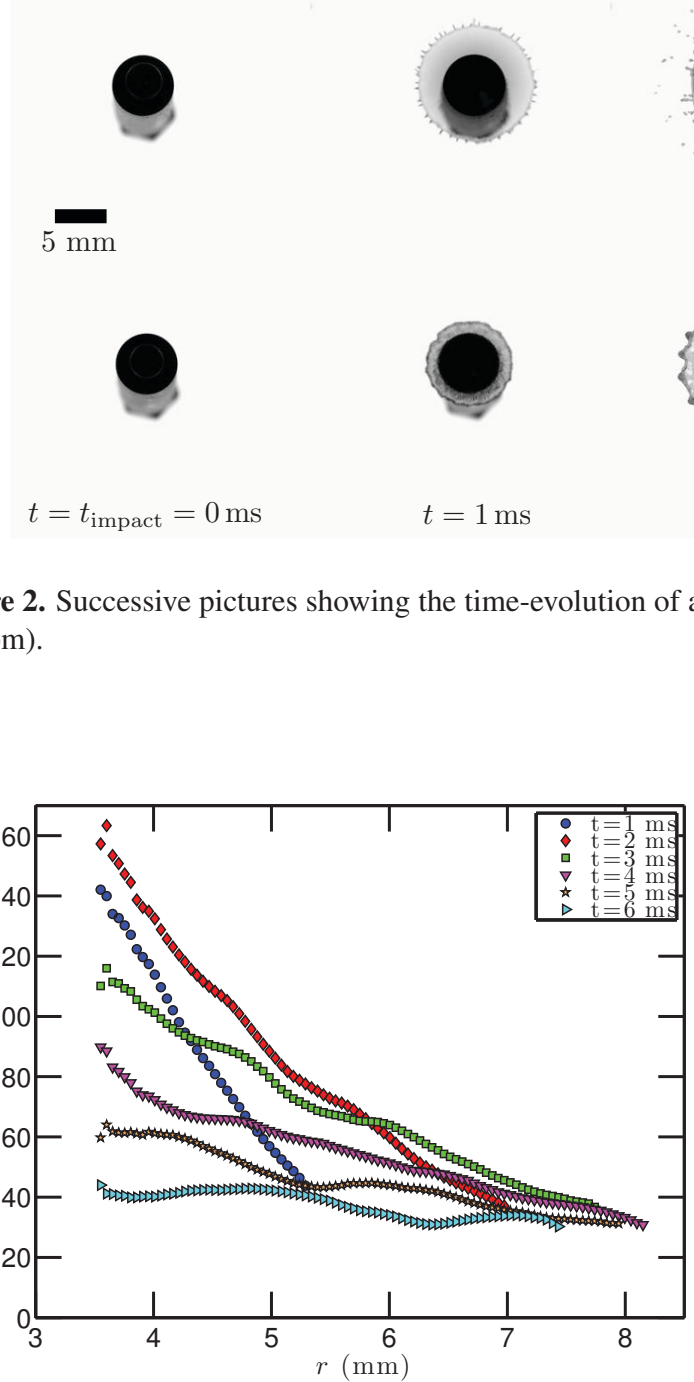

Figure 3. Evolution of the liquid sheet thickness formed by the drop impact of a pure liquid on the target at $t=0 \mathrm{~ms}$ taken at various time. explain these observations: the increase in viscosity due to the addition of particles leads to the change of dynamics. To quantify this influence, we performed similar experiments using water/glycerol mixture that have an effective dynamic viscosity identical to the suspensions. The timeevolution of this Newtonian liquid sheet is shown in Fig. 4 and illustrates that the increase in viscosity induced by the particles cannot account for the differences observed. Indeed, for water/glycerol concentrations corresponding to suspensions with $\phi \leq 20 \%$, no difference is observed. Therefore, the modification of the spreading dynamics is an intrinsic local effect of the particles. Qualitatively, the presence of particles larger than the thickness of the sheet creates a deformation of the interface that limits its expansion and dynamics [11].

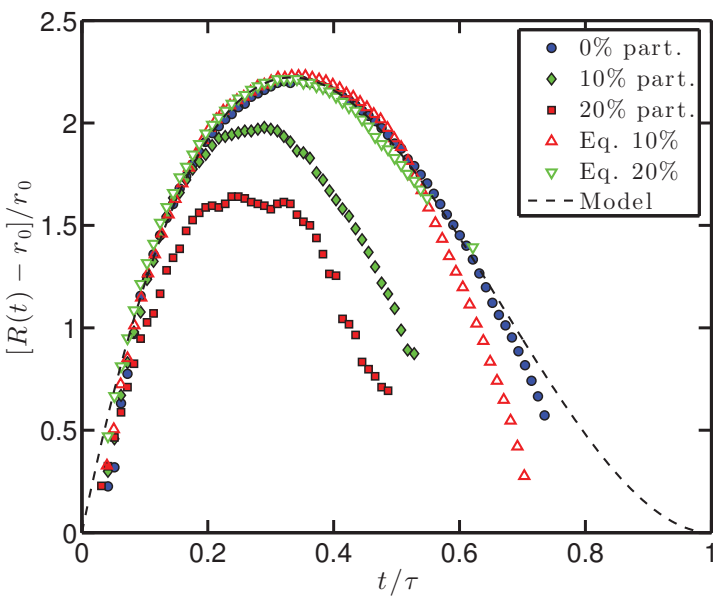

Figure 4. Evolution of the rescaled radius of the liquid sheet as a function of the dimensionless time $t / \tau$ for the pure liquid, suspensions with $10 \% \mathrm{wt}$ and $20 \% \mathrm{wt}$ of particles. The measurements performed with Newtonian liquids of same viscosities as the $10 \%$ and $20 \%$ suspensions are also reported. The dashed-line is the prediction given by eq. (3) with a prefactor $a=15$

\section{Fragmentation of the particle-laden liquid sheet}

During the spreading and the fragmentation of the particleladen liquid sheet, droplets are generated from two different ways. The first mechanism takes place upon impact, during the expansion and the retraction of the liquid film. The droplets are created by the outer rim and have relatively substantial size. Then, droplets are also generated when the liquid film ends its retraction phase and smaller droplets are then created.

We reported in Fig. 5 the resulting droplet size distributions for increasing particles concentration. It shows that the distributions share a similar trend even if they tend to be larger and shift to larger radii as the concentration in particles increases. We also notice that the larger the con- 


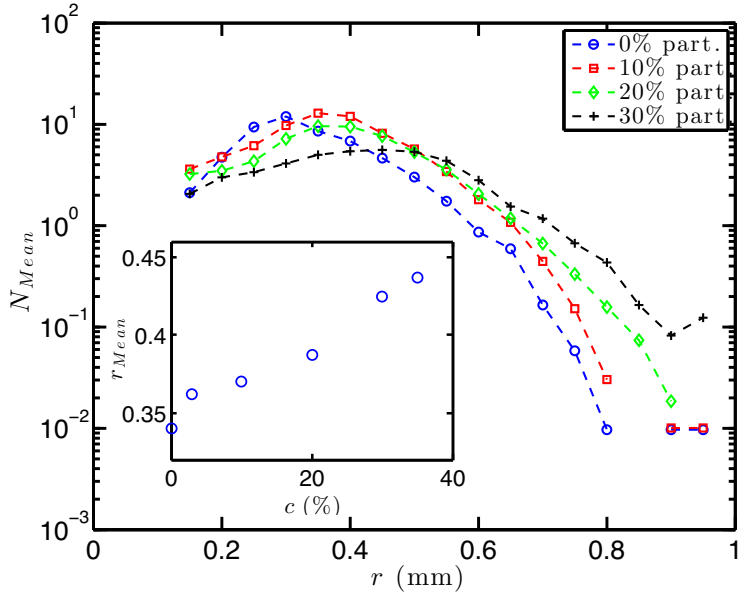

Figure 5. Mean number of droplets per impact $N_{\text {Mean }}$ of radius $r$ for different concentrations of particles. Each curve contains over a hundred drop impacts. Inset: mean radius $r_{\text {Mean }}$ of the droplets resulting from the atomization of the liquid sheet for varying particle concentration $c$.

centration is, the less numerous are the droplets emitted at each impact and these droplets tend to have a large diameter. The evolution of the mean radius $r_{\text {Mean }}$ of the droplets generated during the fragmentation process is plotted in the inset for Fig. 5 as a function of the particle concentration. The mean droplet size increases with the particle concentration.

We have performed the same experiments with Newtonian liquid of increasing viscosity. The viscosities of the solution without particles are taken equal to the viscosity of the different suspensions $(\phi \leq 30 \%)$. We did not notice a significant change in droplet size for Newtonian fluid with similar viscosities. Therefore, the influence of the particles goes beyond the increase in viscosity of the suspension, modifying the generation process of the droplets compared to a classical Newtonian liquid [11].

\section{Conclusion}

In study, we have experimentally studied the formation and atomization of a particle-laden liquid sheet formed upon impact of a drop on a small target. The presence of spherical, neutrally buoyant, non-colloidal particles introduces various effects that make the dynamics of the liquid sheet different from that of a pure liquid.

Our experimental results have first illustrated the influence of the particles on the spreading of the liquid film.
We have characterized the spreading dynamics of the sheet such as its radius and thickness in the presence and absence of particles. We have been able to compare the theoretical prediction for a Newtonian fluids and quantify the deviation to this simple law for a particle-laden liquid sheets.

We have also investigated the destabilization of the liquid sheet and its atomization in droplets with and without particles. Our results suggest that the particles do not alter the physical mechanism of droplet generation, but they modify the mean radius of the drops generated. We have also observed a widening of the distribution and a shift towards larger drop radii when increasing the particles concentration. These effects can not be explained using the effective viscosity and are directly related to the discrete nature of the particles.

Acknowledgements: the authors acknowledge the support from the French ANR (project ProLIFIC ANR-16CE30-0009) and partial support from a CNRS PICS grant $n^{\circ} 07242$.

\section{References}

[1] E. Guazzelli \& J. F. Morris, A physical introduction to suspension dynamics (Cambridge University Press, 2011) $256 \mathrm{pp}$

[2] M. M. Denn \& J. F. Morris, Annu. Rev. Chem. Biomol. Eng. 5, 203-228 (2014)

[3] F. O. Addo-Yobo, M. J. Pitt \& H. A. Obiri, AIChE J. 57(8), 2007-2024 (2011)

[4] R. J. Furbank \& J. F. Morris, Phys. Fluids 16(5), 17771790 (2004)

[5] C. Bonnoit, T. Bertrand, E. Clément \& A. Lindner, Phys. Fluids 24(4), 043304 (2012)

[6] A. Rozhkov, B. Prunet-Foch \& M. Vignes-Adler, Proc. Roy. Soc. A 460(2049), 2681-2704 (2004)

[7] E. Villermaux \& B. Bossa, J. Fluid Mech. 668, 412435 (2011)

[8] B. Keshavarz, E. C. Houze, J. R. Moore, M. R. Koerner, G. H. McKinley, Phys. Rev. Lett. 117(15), 154502 (2016)

[9] C. Vernay, L. Ramos \& C. Ligoure, Phys. Rev. Lett. 115(19), 198302 (2015)

[10] C. Vernay, L. Ramos \& C. Ligoure, J. Fluid Mech. 764, 428-444 (2015)

[11] L. A. Lubbers, Q. Xu, S. Wilken, W. W. Zhang \& H. M. Jaeger, Phys. Rev. Lett. 113(4), 044502 (2014) 